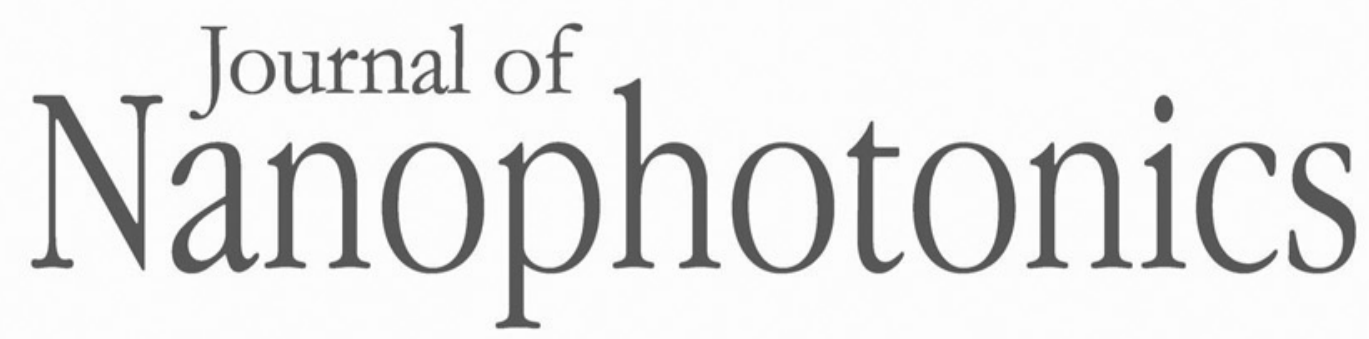

Nanophotonics.SPIEDigitalLibrary.org

\title{
Perfect metamaterial absorber for applications in sustainable and high- efficiency solar cells
}

\author{
Patrick Rufangura \\ Cumali Sabah
}




\title{
Perfect metamaterial absorber for applications in sustainable and high-efficiency solar cells
}

\author{
Patrick Rufangura ${ }^{\mathrm{a}, \mathrm{b}}$ and Cumali Sabah ${ }^{\mathrm{c}, \mathrm{d}}$ 相 \\ ${ }^{a}$ Middle East Technical University-Northern Cyprus Campus, Sustainable Environment and \\ Energy Systems, Kalkanli, Guzelyurt, TRNC/Mersin 10, Turkey \\ ${ }^{b}$ University of Technology Sydney, School of Electrical and Data Engineering, \\ Faculty of Engineering and Information Technology, Ultimo, New South Wales, Australia \\ ${ }^{\mathrm{c}}$ Middle East Technical University—Northern Cyprus Campus, Department of Electrical and \\ Electronics Engineering, Kalkanli, Guzelyurt, TRNC/Mersin 10, Turkey \\ ${ }^{\mathrm{d}}$ Middle East Technical University—Northern Cyprus Campus, Kalkanli Technology Valley, \\ Kalkanli, Guzelyurt, TRNC/Mersin 10, Turkey
}

\begin{abstract}
The current state of energy is characterized by complex challenges in production processes and environmental issues. With the world population continuing to multiply faster and the globalization process, additional energy production is needed to meet future demands. Solar energy is one of the best sustainable energy resources, which is expected to play a vital role in this scenario. One of the best techniques to harvest this resource is through solar photovoltaic technology, which produces electricity directly from solar radiation. But, one of the problems still persisting is its low efficiency. To harness this technology, this problem needs to be addressed. Metamaterial (MTM) technology has enabled the creation of advanced devices for various applications. Solar cell technology is one of the fields to benefit from this technology. MTM perfect absorber can be used in solar cells to improve their absorption. Multiple-bands MTM absorber for next generation high-efficiency solar cells is proposed. The design gives a nearly perfect absorption (99.94\%) with a bandwidth of $23.4 \%$ in visible spectrum. In addition, the geometric flexibility of a proposed design causes its absorption rate to be insensitive of polarization angles and angles of incident electromagnetic radiations. (C) 2018 Society of PhotoOptical Instrumentation Engineers (SPIE) [DOI: 10.1117/1.JNP12.026002]
\end{abstract}

Keywords: metamaterial; perfect absorber; solar cell.

Paper 18007 received Jan. 15, 2018; accepted for publication Mar. 20, 2018; published online Apr. 11, 2018.

\section{Introduction}

The contemporary energy sector is characterized by complex challenges in production processes, economic, geopolitical, and environmental issues. These challenges are available in both industrialized and developing countries but at different levels. continues to multiply faster, which implies that the future energy production needs to be increased to meet the demand. Another concern is the globalization process that is taking place in the present century, where large additional energy supplies are needed to foster the process. In the meantime, predictions on the world's most relied energy production resources, "fossil fuel", shows a decline of this resource for future centuries. ${ }^{\mathbb{Q}}$ With these uncertainties, the only remaining hope is the renewable energy resources and their technological advancement, success in nuclear energy technology (prospective deployment of advanced nuclear reactor systems), and on ability to develop hybrid systems that combine the two resources (nuclear-renewable energy systems) Solar energy is one of the best sustainable energy resources, which is expected to play a vital role in the future energy production, and can be

*Address all correspondence to: Cumali Sabah, E-mail: abah@metuledutt

$1934-2608 / 2018 / \$ 25.00$ (C) 2018 SPIE 
hybridized to nuclear reactors (nuclear-solar power plant) are that it is freely available in all countries (developed and developing countries) across the world, and lots of technologies to harvest this resource have been revealed while others are still coming 10 One of the best techniques to harvest solar energy resources is through solar photovoltaic technology that produces electricity directly from solar radiation using the photoelectric effect. The advantage with this technique is that it can be used for both on grid electricity generation project and off-grid or remote area electrification. But, one of the problems still persisting and hinder solar photovoltaic technology to grow faster is its low efficiency. To harness this technology, this persistent problem needs to be addressed. Currently, there is a great interest in metamaterial (MTM) technology, where an artificial material is made from an already existing substance to display its interesting electromagnetic (EM) properties. 때

EM parameters of MTMs are the permittivity $(\varepsilon)$ and permeability $(\mu)$, which are defined by effective medium theory, and these parameters can be altered through the design of MTMs unit cells. $1 \square$ With this adjustable property, MTMs with $(\varepsilon)$ and $(\mu)$ smaller than that of free space and negative values have been effectively realized for almost all frequency ranges of the solar spectrum. 183 These properties have been enabled in the creation of new advanced devices for various applications in the EM field. the operational wavelength for its properties to be characterized by an effective medium as described by EM theory. Consequently, skillfully designing the MTM unit cell, both its real and imaginary parts of $(\varepsilon)$ and $(\mu)$, might be tailored to the desired standards for them to display the preferred EM properties. This interesting property has enabled the realization of MTM absorbers with the near-perfect absorption response. ${ }^{2}$ The role of tuning $(\varepsilon)$ and $(\mu)$ by adjusting the dimensions of the electric and magnetic resonant components in MTM absorber unit cell enables to match the impedance of the MTM absorber structure to that of free space. As a result, the reflection and transmission coefficient of incident EM radiations can be reduced to the minimum possible values at the resonant frequency causing a higher absorption rate. The first MTM absorber was realized by Landy et al ${ }^{2}$ The unit cell of their MTM absorber consists of metallic ring resonator, split wire, and a dielectric spacer. At certain conditions, by carefully tuning the resonators geometry, their structure's impedance matched to the free space and they achieved absorption of $98 \%$ at $1.12 \mathrm{THz}$ and $70 \%$ at $1.3 \mathrm{THz}$, from numerical simulation and experiment, respectively. Since then, the designs and characterization of MTM absorber have obtained a significant interest. For example, Kajtar et al ${ }^{-3}$ theoretically proposed a single, dual, and triple homogeneous metal-insulator-metal absorber with absorption rate of about a unit in visible frequency regions, although their absorption was found to be sensitive of TE and TM polarization angles. Liu et al $\$$ experimentally and theoretically reported a dual-band MTM perfect absorber based on artificial molecules with near $100 \%$ narrow absorption in microwave frequencies. Zhang et al 23 proposed a dual-band MTM absorber with $95 \%$ absorption in visible frequency regimes. Kim et al ${ }^{3}$ reported a triple-band MTM absorber in $\mathrm{GHz}$ frequencies. Moreover, numerous MTM absorber structures were also proposed, characterized, and fabricated for potential applications in microwave, 3 terahertz, frequency ranges. Though with high absorptivity, most of the existing MTM absorbers are polarization dependent and narrow accepted angles, which limit their potential applications. In addition, little work has been done for MTM absorbers in the visible frequencies compared to other frequency ranges, yet, solar radiations peak in this range. 5 Consequently, much work is still needed to be done for MTM absorbers operating in the visible region to collect solar energy effectively.

It is believed that efficiency of solar cells can be improved if a near-perfect absorber based on MTM is used in the place of the actual solar cells' absorber $\sqrt{2}$ This study presents a multipleband MTM perfect absorber for next generation high-efficiency solar cell technology. The proposed design offers a near-perfect absorption rate $(99.94 \%)$ in visible spectra regime (469.74 nm, see Fig. (2) with an outstanding absorption bandwidth of $23.4 \%$ with respect to the resonant frequency. Additionally, the geometric flexibility characterizes the proposed design confirmed by its ability to offer constant near-perfect absorption over a wide incident angles and for TE and TM polarized radiations. 


\section{Simulation of a Multiband Metamaterial Absorber Design}

Efficiency of absorber is described as $A(\omega)=1-[R(\omega)+T(\omega)]$, where $A(\omega), R(\omega)$, and $T(\omega)$ are frequency-dependent absorption, reflection, and transmission rate, respectively. It is obvious that the highest absorption attained when reflection and transmission rates are simultaneously minimized to the very minimum possible values. To exclude transmission $[T(\omega)=0]$, it is recommended to use a metal layer as a ground plane $\mathrm{g}$ Matching the impedance of an MTM absorber to the one for free space is an important step to guarantee that the incident radiation is coupled to the absorber with minimum reflection. If one needs to design a multiband MTM with near-perfect absorption, the geometric parameters and shape of unit cell need to be well selected and optimized to make sure that the impedance of free space is about one at every resonant frequency.

The proposed multiband MTM absorber unit cell is shown in Fig. [a] with its geometry parameters. The MTM absorber comprises of three layers. The topmost layer consists of an array of patches (combination of aluminum and alumina layers Ref. 60), which are mainly accountable for the electric response to the incident field. The intermediate layer is an alumina $\left(\mathrm{Al}_{2} \mathrm{O}_{3}\right)$ layer, and the bottom layer is aluminum, which is employed to block the transmission and exciting antiparallel surface current at the corresponding region. Coupling of bottom layer with the dielectric spacer produces the magnetic response. The optical properties of the materials used are defined as frequency-dependent complex parameters and the required parameters are taken from Refs. 60-62 in which they are described as Drude-Lorentz materials.

The numerical simulation is performed with the use of a full-wave EM simulation package, which uses a finite integration technique. MTM absorber intended to operate in visible wavelength regions. The incident waves polarize in a way that the magnetic $(H)$ and electric $(E)$ fields propagate in parallel with the structure plane, with wave vector $(k)$ perpendicular to the geometric plane of the structure. The boundary conditions are fixed to periodic boundaries along $X$ - and $Y$-directions, whereas open and add space boundaries are used for the simulation environment ( $Z$ plane).
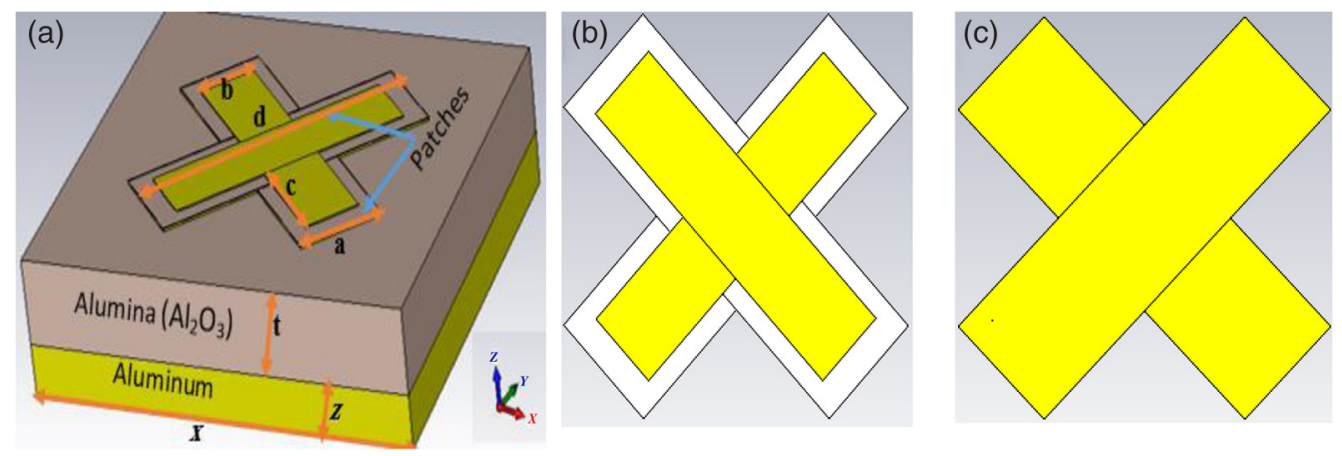

$(d)$

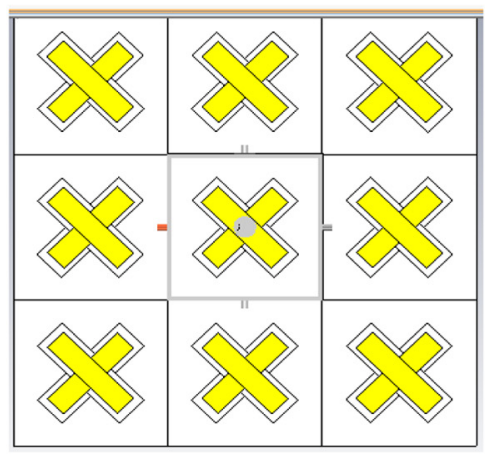

Fig. 1 Perspective view of the proposed MTM absorber structure: (a) unit cell and its geometry parameters, (b) front view of the patches, (c) back view of the patches, and (d) a full structure of the proposed MTM absorber. 


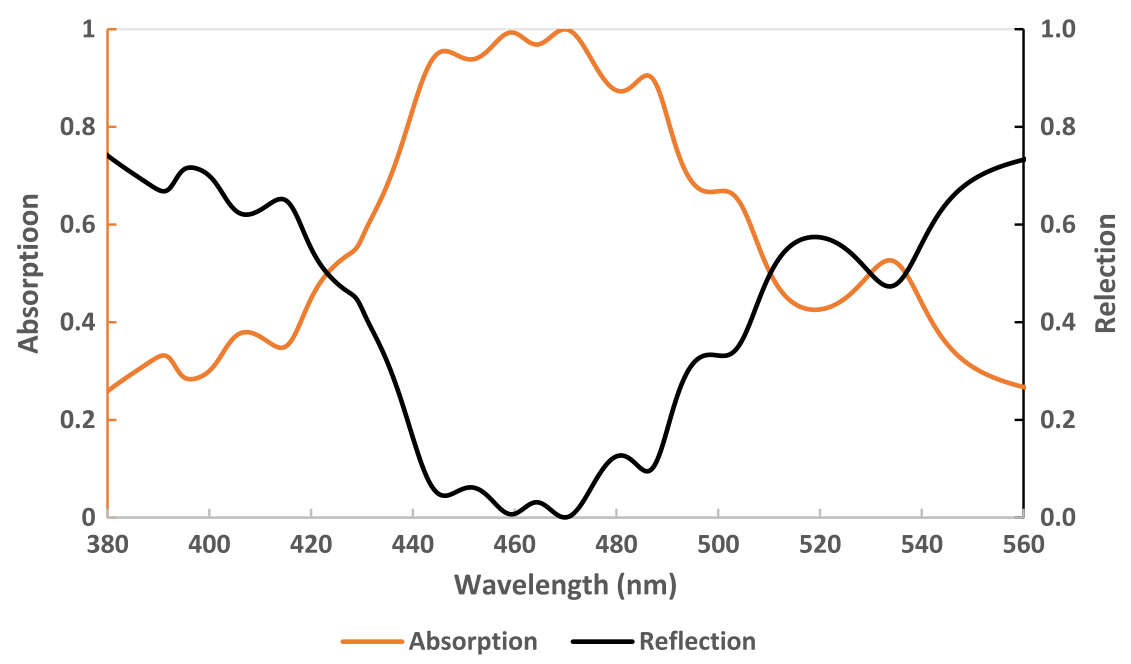

Fig. 2 Simulated absorption and reflection characteristics of the proposed MTM absorber.

The unit cell geometry parameters are as follow: $a=130 \mathrm{~nm}, b=86.7 \mathrm{~nm}, c=185.7 \mathrm{~nm}$, $d=433.3 \mathrm{~nm}, t=111.6 \mathrm{~nm}, z=70 \mathrm{~nm}, x=580 \mathrm{~nm}$, and the thickness of each patch is $3 \mathrm{~nm}$.

Since the bottom metal of thickness greater than its skin depth 10 因国 was employed (i.e., transmission is zero), the total absorption will be calculated as $A(\omega)=1-R(\omega)$. In Fig. Q the simulated spectra absorption and reflectance are depicted as function of wavelength. Four peaks absorption was observed at $446.50,459.41,469.74$, and $485.43 \mathrm{~nm}$ wavelengths with an absorption rate of $95.04 \%, 99.39 \%, 99.95 \%$, and $90.75 \%$, respectively.

The four peak bands have an absorption bandwidth of $23.4 \%$, in which absorption rate is $>90 \%$. The origin of the four peaks is believed to be the four sides of the patches (X-shape), where each side corresponds to absorption resonant.

To understand the physics behind the absorption mechanism of the proposed absorber, electric field distributions at the surface of patches and surface current distributions on the metal for incident EM wave are analyzed for the four resonant wavelengths. The top of the patches is irradiated by incident E-field in such a way that opposite charges are accumulated on the four sides of the patches. These are explained by the four different directions of electric field observed at the edges of the patches. In Fig. 3, electric field distributions (amplitude) are reported for four peaks absorption. On the other hand, the current flows at the surface of metal are strongly located at the edges of the metal. In this case, the patches work as multipoles/quadrupoles. Surface current distributions for all resonant wavelengths are also plotted in Fig. $⿴$.

As one can notice in Fig. $甘$, both parallel and antiparallel surface currents exist at the surface of metallic patch, which indicate the existence of magnetic flux among the patches. The absorber acts together with the incident EM field resulting in EM resonances. At this instant, EM parameters are matched to that of free space and then high-energy absorption is attained. 90 田

To comprehend the effect of the geometry parameters on the absorption characteristics of the proposed MTM absorber, parametric study is discussed. In Fig. 5, the simulation results under different geometry parameters are shown. First, numerical simulation is performed for different dielectric spacer thickness " $t$." The dielectric thickness is altered from $t=90$ to $126 \mathrm{~nm}$ with a step width of $9 \mathrm{~nm}$ [see Fig. 5(a)]. As one can observe in Fig. 5(a), the resonant wavelength shifts toward the left (higher wavelengths) as the dielectric thickness increases. This character is very important, for the proposed structure, since it can be implemented in solar to operate in a wide range of wavelengths and still offer outstanding absorption responses.

A second investigation is conducted on the impact of the patches' dimension to their effect of the absorption coefficient of the proposed MTM absorber. Beginning with parameter " $d$," as shown in Fig. 5(b), where by changing its dimension around the reference value $(d=$ $433.3 \mathrm{~nm}$ ) different absorption was noticed. As one can see in Fig. 5(b), increasing $d$ causes reduction of total absorption gain by the MTM absorber. The largest $d(d=490.6 \mathrm{~nm})$ 

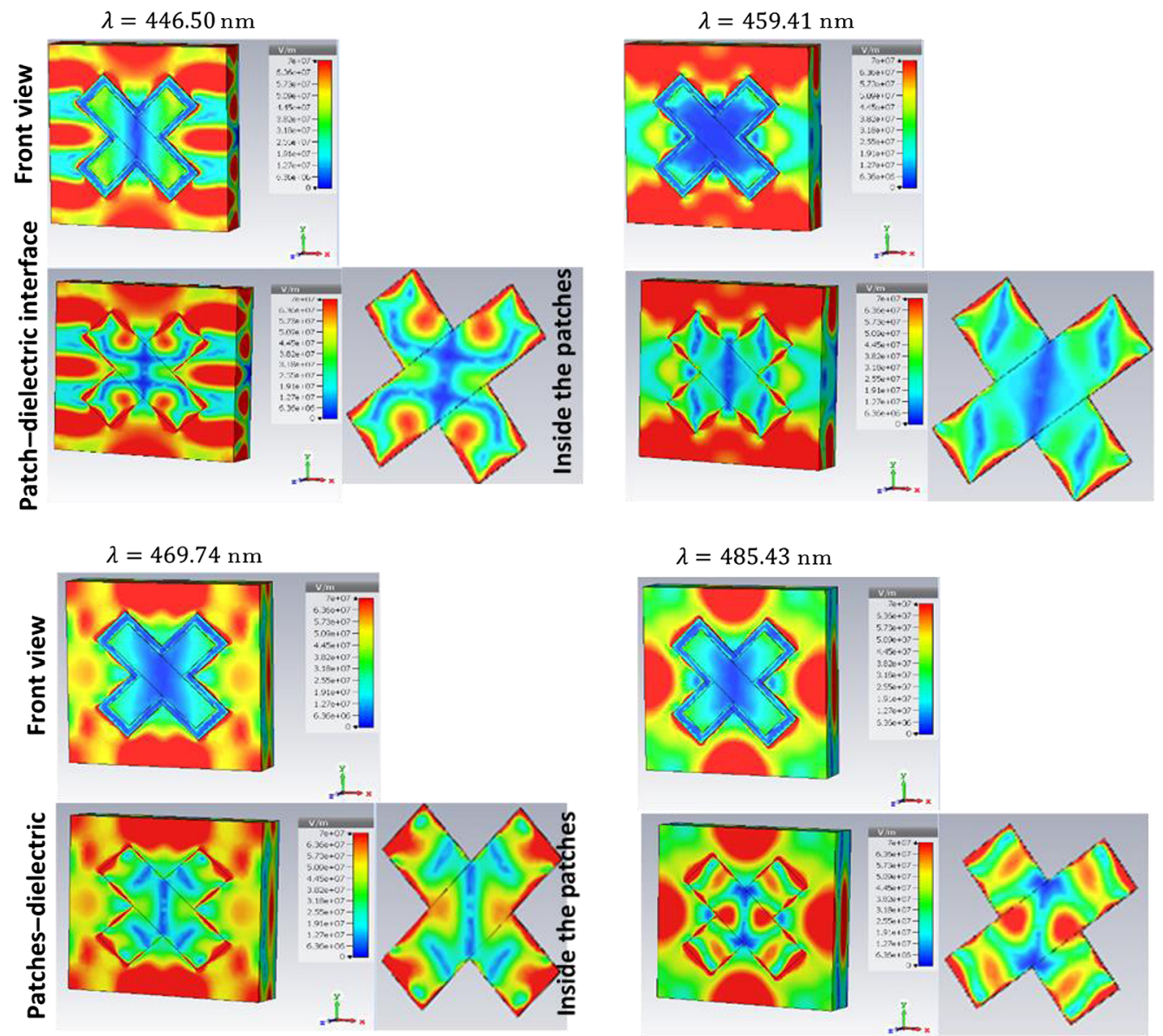

Fig. 3 Electric field distributions of the proposed MTM absorber for four different resonant wavelengths: $446.50,459.41,469.74$, and $485.43 \mathrm{~nm}$ (viewed from the top, at the interface of dielectric-patches and inside the patches).
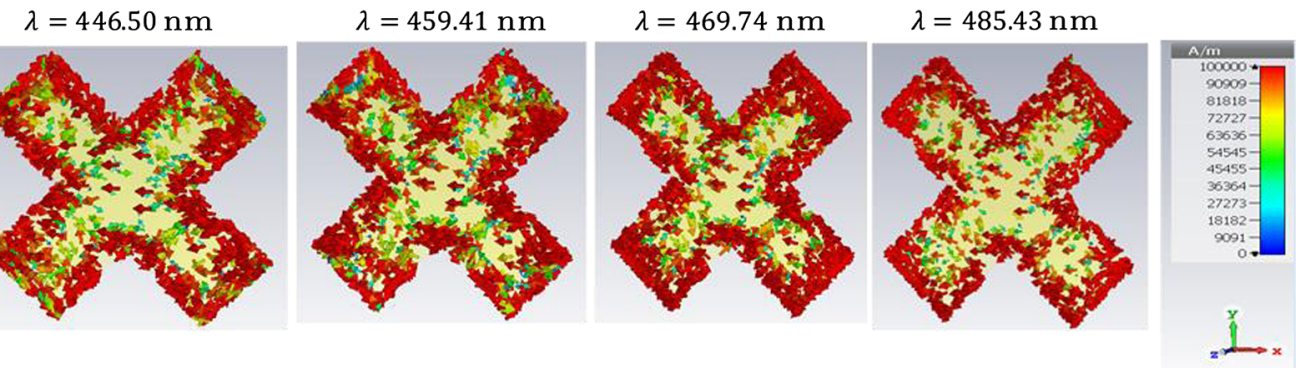

Fig. 4 Surface current distributions of the proposed MTM absorber for four different resonant wavelengths: $446.50,459.41 \mathrm{~nm}, 469.74$, and $485.43 \mathrm{~nm}$.

corresponds to the least absorption rate. On the other hand, decreasing $d$ enables the optimization of the total absorption gain for an MTM absorber proposed in this work, where the best absorption obtained at $d=412.7 \mathrm{~nm}$ [the ripples are eliminated and a wideband with perfect absorption is gained, see green curve in Fig. [(b)]. Another patch's parameter that plays an important role on the absorptivity of the proposed MTM absorber is " $a$." By varying the dimensions of " $a$," absorption characteristics of the proposed MTM absorber change accordingly, where the best absorption is achieved at small value, $a=92.9 \mathrm{~nm}$ (the absorption of the proposed structure turn out to be wider and higher at this dimension). As it can be seen in Fig. 5(c), the absorption band of the proposed design tends to become narrower as the dimension of " $a$ " is increasing. 
Rufangura and Sabah: Perfect metamaterial absorber for applications in sustainable...
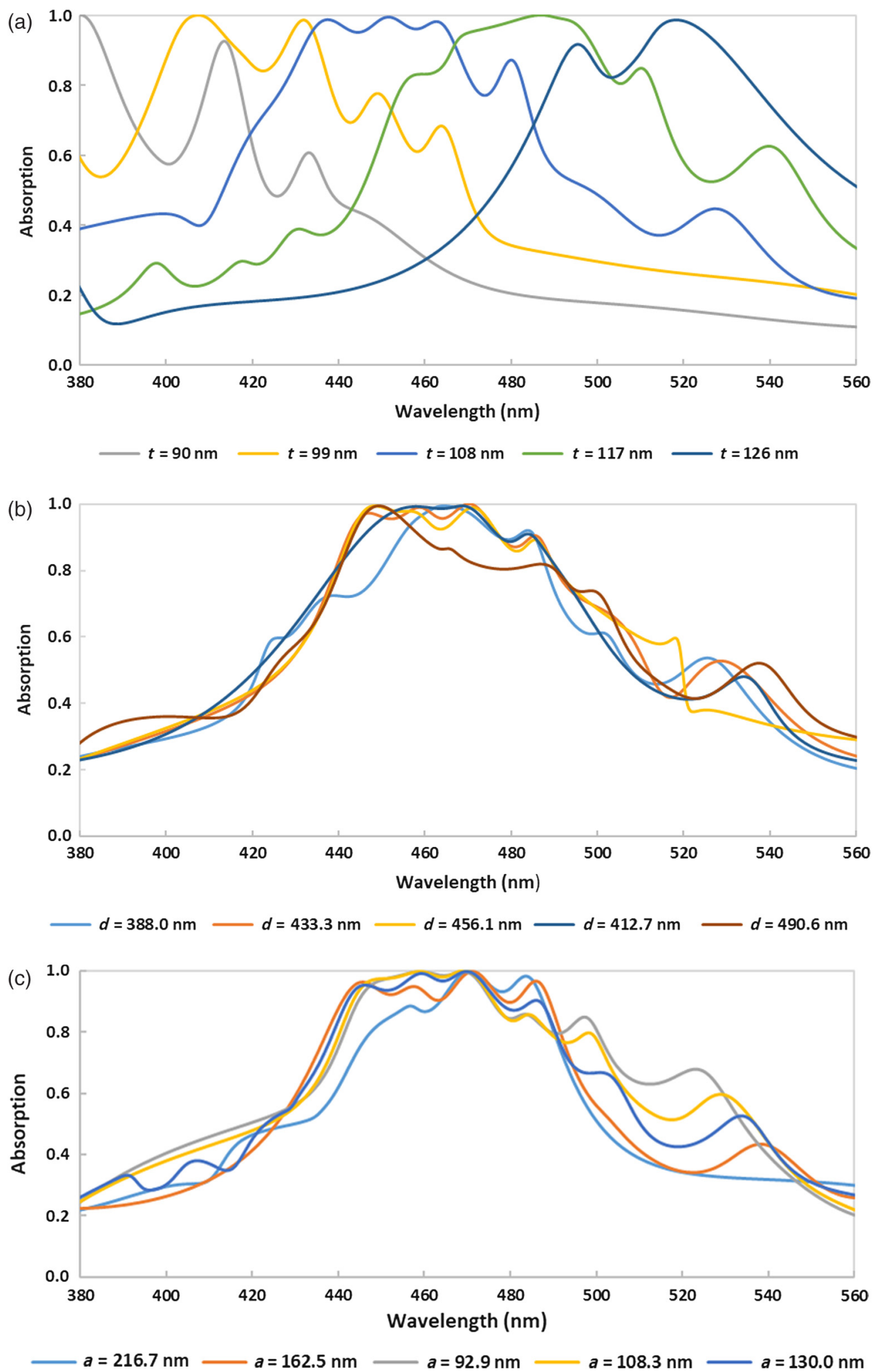

Fig. 5 Simulated absorption results for the proposed MTM absorber at different geometry parameter: (a) different dielectric thickness " $t$;" patches' dimensions (b) different value of " $d$," and (c) different value of "a." 
Rufangura and Sabah: Perfect metamaterial absorber for applications in sustainable...
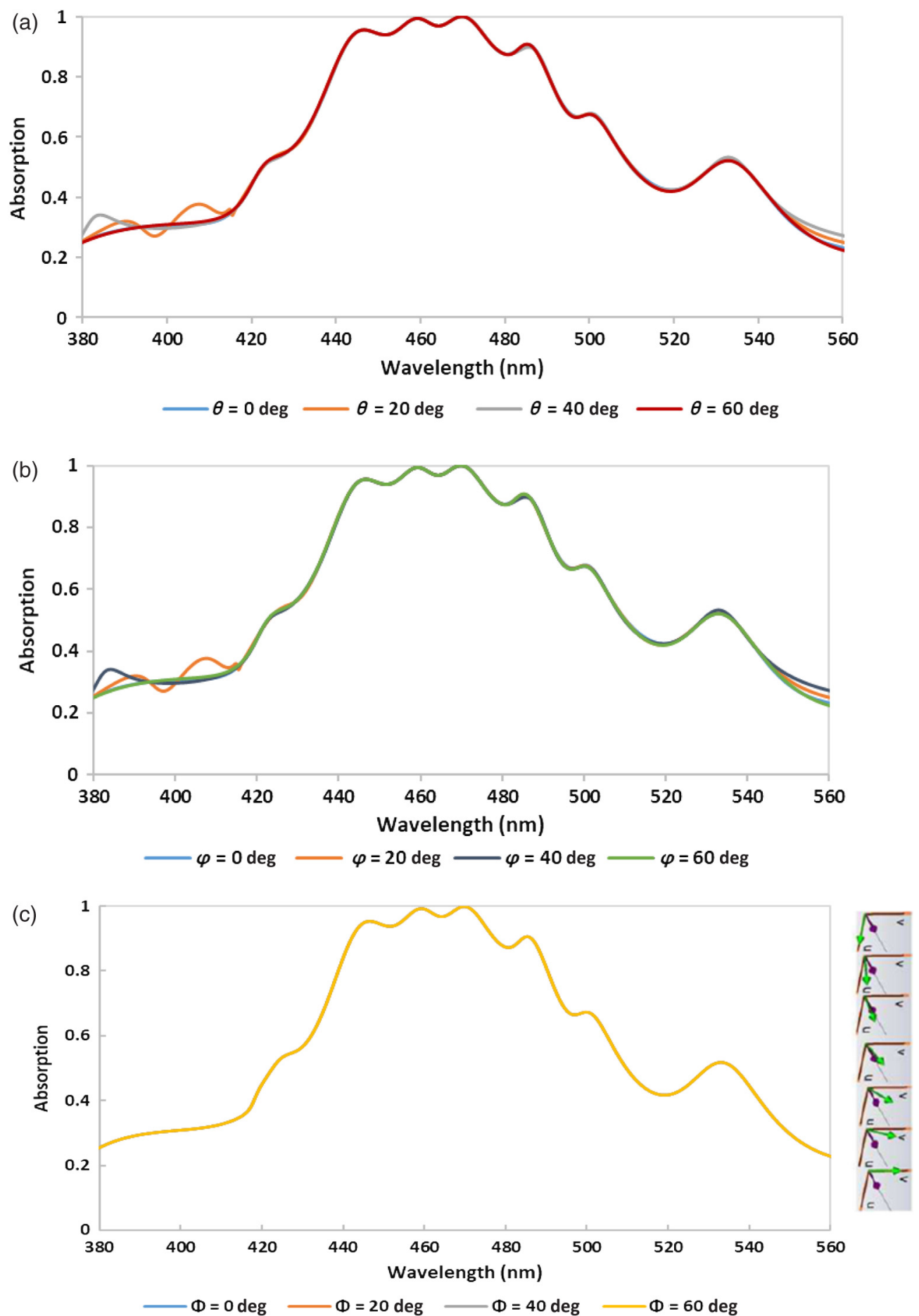

Fig. 6 Simulated absorption characteristics of the proposed MPA structure under different polarization angles and angles of incident EM radiations: (a) TE polarization ( $\theta$ ), (b) TM polarization $(\varphi)$, and (c) different $(\Phi)$ of incident angle.

Thus, if one wants this structure to produce a wideband perfect absorption, its " $a$ " dimension must be about $90 \mathrm{~nm}$.

Moreover, to understand the absorptivity of the proposed MTM absorber with respect to the source of EM radiations, a study is conducted for different polarization angles for TE and TM polarized waves and under different incident angles. Figure 6 shows the simulated results for different polarization angles for TM and TE waves and different incident angles for EM radiations. The polarization angle is rotated from $0 \mathrm{deg}$ to $60 \mathrm{deg}$ with a step of $20 \mathrm{deg}$. As shown in 
Figs. 6(a) 6(b), the absorption response of the MTM absorber in the present study shows polarization angle independency for both TE and TM polarized radiations. Note that the polarization angle can be defined as the angle variation on $x-y$ plane (see Fig. 1) for TE and TM wave cases (E-field component of the polarization wave rotating on $x-y$ plane).

In addition, as shown in Fig. 6(c), the proposed design offers perfect absorption for incident angles of EM radiations (see the figure inset, the rotation in the green vector). This is found by conducting numerical simulation on the proposed MTM absorber unit cell at different incident angles. By changing incident angles from 0 deg to $60 \mathrm{deg}$ with step width of $20 \mathrm{deg}$, the absorption rate was found to be uniform for all incident angles. The above behaviors confirm the symmetric character of the structure under study. The absorbers of this kind are outstanding candidates for the realization of high absorber devices working in the visible spectrum regimes and can be employed for the creation of high-efficiency solar cells since the absorption of the solar cells can remain high irrespective of the angle of polarization for both TE and TM polarized waves and for wide incident angles.

\section{Conclusions}

Near-perfect absorbers based on MTM were proposed and numerically described in this paper. The wavelength response for the proposed MTM absorber produces multiple-band absorption resonances with nearly perfect absorption (99\%) rate, which rose from the strong EM resonance that produced by robust coupling between patches-dielectric spacer and patches-ground plane. The geometry parameters of the design are investigated and considered for their effect on the absorption properties of the proposed structure and a wideband absorption response was revealed as a result of the structure geometric optimization. Additional analysis was performed on electric fields and surface currents distributions for understanding of the physics and the absorption characteristics of the proposed absorber. Finally, a study directed on absorption capabilities of the proposed MTM absorber at different incident angles for EM radiations prove the structure to be incident angles and polarization angles insensitive for TE and TM polarized radiations. The proposed MTM structure can be used for creation of high absorber solar cells in visible frequencies. The experimental work of this design remains a subject for future study.

\section{Acknowledgments}

P. Rufangura acknowledges the financial support of the Government of Rwanda for his graduate studies at Middle East Technical University-Northern Cyprus Campus (METU-NCC) through the Rwanda Education Board (REB), Sponsorship No. 911/12.00/SR/2013. The work reported here was carried out at METU-NCC. It was supported by METU-NCC under the Grant Nos. BAP-FEN-15-D-3 and BAP-FEN-16-K-8.

\section{References}

1. REN21, "Renewables 2014 global status report," REN21 Secretariat, Paris, http://www ren21|.net/Portals/0/documents/Resources/GSR/2014/GSR2014_KeyFindings_low\%20res. pdf (2014).

2. World Energy Council, "World energy resources 2013 survey," London, https://www, worldenergy/org/wp-content/uploads/2013/09/Complete_WER_2013_Surveylpdf.

3. World Economic Forum, "Global energy architecture performance index report," Cologny/ Geneva, Switzerland, http://www3/.weforum.org/docs/WEF_GlobalEnergyArchitecture_ 2015.pdf (2015).

4. International Energy Agency, "World energy outlook 2014," https://wwwiea.org/ publications/freepublications/publication/WEO2014.pdf.

5. Organization for Economic Co-Operation and Development, "Energy: the next fifty years," OECD publications, Paris, France, https://www/oecd.org/futures/17738498.pdf (1999). 
6. J. Twidell and T. Weir, Renewable Energy Resources, 2nd ed., Taylor \& Francis, Oxon, United Kingdom (2006).

7. N. Victor, "Technology perspectives from 1950 to 2100 and policy implications for the global nuclear power industry," Prog. Nucl. Energy 105, 83-98 (2018).

8. S. Siddharth, "Hybrid nuclear-renewable energy systems: a review," t. Cleaner Prod. 181, 166-177 (2018).

9. M. F. Keller, "Hybrid integrated energy production process," US Patent No. 7,961,835 (2011).

10. B. Parida, S. Iniyan, and R. Goic, "A review of solar photovoltaic technologies," Renewable Sustainable Energy Rev. 15, 1625-1636 (2011).

11. T. T. Chow, "A review on photovoltaic/thermal hybrid solar technology," Appl. Energy 87, 365-379 (2010).

12. G. K. Singh, "Solar power generation by PV (photovoltaic) technology: a review," Energy 53, 1-13 (2013).

13. J. M. Hernández et al., "An overview of solar photovoltaic energy in Mexico and Germany," Renewable Sustainable Energy Rev. 31, 639-649 (2014).

14. A. Sihvola, "Metamaterials in electromagnetic," Metamaterials 1, 2-11 (2007).

15. V. G. Vesalago and P. N. Labedev, "The electrodynamics of substances with simultaneously negative values of $\varepsilon$ and m," Sov. Phys. Usp. 10, 509-514 (1968).

16. N. Engheta and R. W. Ziolkowski, Metamaterials, Physics and Engineering Explorations, John Wiley \& Sons Inc., IEEE Press, Hoboken, New Jersey (2006).

17. B. Zhu et al., "Polarization insensitive metamaterial absorber with wide incident angle," Prog. Electromagn. Res. 101, 231-239 (2010).

18. C. Caloz and T. Itoh, Electromagnetic Metamaterials: Transmission Line Theory and Microwave Applications, Wiley, New York (2006).

19. M. Gokkavas et al., "Experimental demonstration of a left-handed metamaterial operating at 100 GHz," Phys. Rev. B 73, 193103-193106 (2006).

20. S. Zhang et al., "Experimental demonstration of near-infrared negative-index metamaterials," Phys. Rev. Lett. 95, 137404-137407 (2005).

21. D. Schurig et al., "Metamaterial electromagnetic cloak at microwave frequencies," Science 314, 977-980 (2006).

22. S. Linden et al., "Magnetic response of metamaterials at 100 terahertz," Science 306, 13511353 (2004).

23. J. Yao et al., "Optical negative refraction in bulk metamaterials of nanowires," Science 321, 930-930 (2008).

24. J. B. Pendry, "Perfect cylindrical lenses," Opt. Express 11(7), 755-760 (2003).

25. F. Zolla et al., "Electromagnetic analysis of cylindrical invisibility cloaks and the mirage effect," Opt. Lett. 32, 1069-1071 (2007).

26. C. Wu et al., "Metamaterial-based integrated plasmonic absorber/emitter for solar thermophotovoltaic systems," V. Opt. 14, 024005 (2012).

27. A. Savin et al., "An electromagnetic sensor with a metamaterial lens for non-destructive evaluation of composite materials," Sensors 15, 15903-15920 (2015).

28. Y. Luo et al., "New concept conformal antennas utilizing metamaterial and transformation optics," IEEE Antennas Wirel. Propag. Lett. 7, 509-512 (2008).

29. N. I. Landy et al., "A perfect metamaterial absorber," Phys. Rev. Lett. 100, 207402 (2008).

30. G. Kajtar et al., "Theoretical model of homogeneous metal-insulator-metal perfect multi-band absorbers for the visible spectrum," J. Phys. D Appl. Phys. 49(5), 055104 (2016).

31. X. Liu et al., "Dual band metamaterial perfect absorber based on artificial dielectric 'molecules',' Sci. Rep. 6, 28906 (2016).

32. Z. Zhang et al., "Dual-band nearly perfect absorber at visible frequencies," Opt. Mater. Express 8(2), 463-468 (2018).

33. Y. J. Kim et al., "Triple-band metamaterial absorber based on single resonator," Curr. Appl. Phys. 17(10), 1260-1263 (2017).

34. L. Li, Y. Yang, and C. Liang, "A wide-angle polarization-insensitive ultra-thin metamaterial absorber with three resonant modes," J. Appl. Phys. 110, 063702 (2011). 
35. G. D. Wang et al., "Polarization-insensitive triple-band microwave metamaterial absorber based on rotated square rings," Prog. Electromagn. Res. 145, 175-183 (2014).

36. B. Wang, T. Koschny, and C. M. Soukoulis, "Wide-angle and polarization-independent chiral metamaterial absorber," Phys. Rev. B 80, 033108 (2009).

37. J. Sun et al., "An extremely broad band metamaterial absorber based on destructive interference," Opt. Express 19, 21155-21162 (2011).

38. J. W. Park et al., "Multi-band metamaterial absorber based on the arrangement of donut-type resonators," Opt. Express 21, 9691-9702 (2013).

39. Q. Y. Wen et al., "Terahertz metamaterials with $\mathrm{VO}_{2}$ cut-wires for thermal tunability," Appl. Phys. Lett. 97, 021111 (2010).

40. H. Tao et al., "Highly flexible wide angle of incidence terahertz metamaterial absorber: design, fabrication, and characterization," Phys. Rev. B 78, 241103 (2008).

41. H. Tao et al., "A metamaterial absorber for the terahertz regime: design, fabrication and characterization," Opt. Express 16, 7181-7188 (2008).

42. X. Shen et al., "Triple-band terahertz metamaterial absorber: design, experiment, and physical interpretation," Appl. Phys. Lett. 101, 154102 (2012).

43. F. Dincer et al., "Polarization angle independent perfect metamaterials absorbers for solar cell applications in the microwave, infrared and visible regime," Prog. Electromagn. Res. 144, 93-101 (2014).

44. P. Rufangura and C. Sabah, "Dual-band perfect metamaterial absorber for solar cell applications," Vacuum 120, 68-74 (2015).

45. P. Rufangura and C. Sabah, "Polarization angle insensitive dual-band perfect metamaterial absorber for solar cell applications," Phys. Status Solidi Q 12, 1241-1245 (2015).

46. P. Rufangura and C. Sabah, "Design and characterization of a novel dual-band perfect metamaterial absorber for solar cell applications," f. Alloys Compd. 671, 43-50 (2016).

47. X. Duan et al., "Polarization-insensitive and wide-angle broadband nearly perfect absorber by tunable planar metamaterials in the visible regime," J. Opt. 16, 125107 (2014).

48. Z. Li, B. Serkan, and A. Koray, "Large-area, lithography-free super absorbers and color filters at visible frequencies using ultrathin metallic films," ACS Photonics 2(2), 183-188 (2015).

49. P. Rufangura and C. Sabah, "Wide-band polarization independent perfect metamaterial absorber based on concentric rings topology for solar cells application," t. Alloys Compd. 680, 473-479 (2016).

50. A. Ghobadi et al., "Tuning the metal filling fraction in metal-insulator-metal ultrabroadband perfect absorbers to maximize the absorption bandwidth," Photonics Res. 6(3), 168-176 (2018).

51. M. Chirumamilla et al., "Multilayer tungsten-alumina-based broadband light absorbers for high-temperature applications," Opt. Mater. Express 6(8), 2704-2714 (2016).

52. P. Rufangura and C. Sabah, "Theoretical and thermal characterization of a wideband perfect absorber for application in solar cells," Appl. Phys. A 122(12), 995 (2016).

53. H. Deng et al., "Broadband perfect absorber based on one ultrathin layer of refractory metal," Opt. Lett. 40(11), 2592-2595 (2015).

54. A. Ghobadi et al., "Ultra-broadband, wide angle absorber utilizing metal insulator multilayers stack with a multi-thickness metal surface texture," Sci. Rep. 7, 4755 (2017).

55. K. Aydin et al., "Broadband polarization-independent resonant light absorption using ultrathin plasmonic super absorbers," Nat. Commun. 2, 517 (2011).

56. R. A. Pala et al., "Omnidirectional and broadband absorption enhancement from trapezoidal Mie resonators in semiconductor metasurfaces," Sci. Rep. 6, 31451 (2016).

57. P. Rufangura and C. Sabah, "Graphene-based wideband metamaterial absorber for solar cells application," V. Nanophotonics 11(3), 036008 (2017).

58. P. Rufangura and C. Sabah, "Polarisation insensitive tunable metamaterial perfect absorber for solar cells applications," EET Optoelectron. 10(6), 211-216 (2016).

59. B. Mulla and C. Sabah, "Perfect metamaterial absorber design for solar cell applications," Waves Random Complex Media 25, 382-392 (2015).

60. E. D. Palik, Handbook of Optical Constant of Solids, Academic Press, San Diego, California (1985). 
61. M. A. Ordal et al., "Optical properties of the metals $\mathrm{Al}, \mathrm{Co}, \mathrm{Cu}, \mathrm{Au}, \mathrm{Fe}, \mathrm{Pb}, \mathrm{Ni}, \mathrm{Pd}, \mathrm{Pt}, \mathrm{Ag}$, Ti, and W, in the infrared and far infrared," Appl. Opt. 22, 1099-1120 (1983).

62. P. W. Gilberd, "The anomalous skin effect and the optical properties of metals," J.Phys. $P$ Met. Phys. 12, 1845-1860 (1982).

Patrick Rufangura received his BSc and MSc degrees in applied physics and in sustainable environment and energy systems, respectively. Currently, he is a $\mathrm{PhD}$ research student at the School of Electrical and Data Engineering, University of Technology Sydney, Australia. His current research interests focus on hetero-epitaxial growth, fabrication and optical characterization of graphene on silicon carbide micro/nanostructures on silicon substrates, and metamaterial (MTM) absorbers.

Cumali Sabah received his BSc, MSc, and PhD degrees in electrical and electronics engineering. Currently, he is an associate professor in the Electrical and Electronics Engineering Department and an assistant to the president at Middle East Technical University-Northern Cyprus Campus (METU-NCC). His research interests include the microwave and electromagnetic investigation of unconventional materials and structures, wave propagation, scattering, complex media, MTMs and their applications, and solar systems. 